\title{
Drinking cow's milk alters vitamin D and iron stores in young children
}

New findings suggest that in most young children, two cups of cow's milk per day is sufficient to maintain healthy levels of vitamin $\mathrm{D}$ without having a negative effect on iron stores.

Many international organizations recommend that children drink milk to promote bone health. However, cow's milk is thought to have a

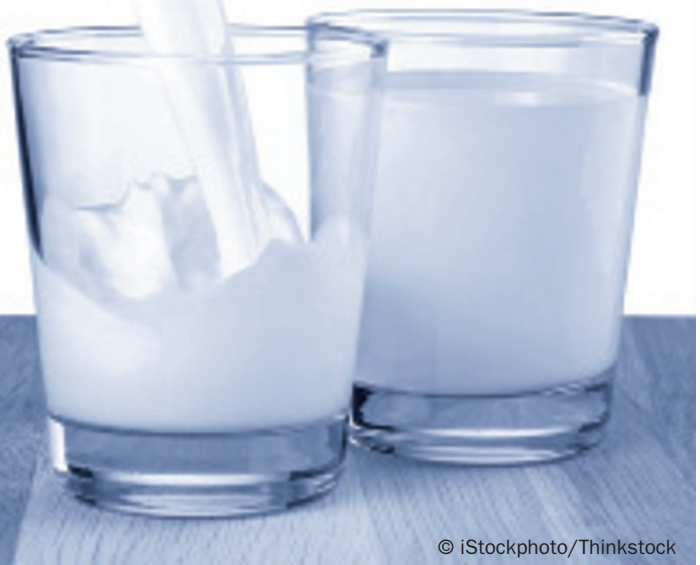

negative effect on iron stores in young children. The effect of different levels of milk consumption on stores of vitamin D and iron have now been examined.

A parent-completed data-collection form was used to gather demographic and nutritional information for 1,311 children aged 2-5 years from Toronto, Canada. Blood samples were also taken to measure serum levels of 25-hydroxyvitamin D and ferritin. Weight, height and skin pigmentation were also measured.

The researchers found that as consumption of cow's milk increased, levels of 25-hydroxyvitamin D also increased, but levels of ferritin decreased. Each additional $250 \mathrm{ml}$ cup of cow's milk increased 25-hydroxyvitamin D levels by $6.5 \%$ and reduced ferritin levels by $3.6 \%$. Vitamin D supplementation, light skin pigmentation, summer season and lower BMI increased levels of 25-hydroxyvitamin $\mathrm{D}$, and winter season increased levels of ferritin.
The authors of the paper suggest that these findings indicate that a trade-off exists between increasing 25-hydroxyvitamin D levels and decreasing ferritin levels with increasing consumption of cow's milk. "On average, $\sim 2$ cups ( $500 \mathrm{ml}$ ) of cow's milk per day was sufficient to maintain median 25-hydroxyvitamin $\mathrm{D}>75 \mathrm{nmol} / \mathrm{l}$ with minimal impact on median serum ferritin," write the authors. However, the authors point out that children with darker skin pigmentation not receiving vitamin $\mathrm{D}$ supplements during the winter require 3-4 cups of milk a day to achieve optimal levels of 25-hydroxyvitamin $\mathrm{D}$, which could decrease levels of ferritin.

\section{Claire Greenhill}

Original article Maguire, J. L. et al. The relationship between cow's milk and stores of vitamin D and iron in early childhood. Pediatrics 131, e144-e151 (2013) 